Lynn M. Bekris, PhD

Associate Staff, Genomic Medicine Institute

Lerner Research Institute, Cleveland Clinic

Assistant Professor of Molecular Medicine,

Cleveland Clinic Lerner College of Medicine of

Case Western Reserve University, Cleveland, $\mathrm{OH}$
James B. Leverenz, MD

Director, Cleveland Lou Ruvo Center for Brain

Health, Neurological Institute, and Joseph Hahn, MD,

Endowed Chair of the Cleveland Clinic Neurological

Institute, Cleveland Clinic

\title{
Emerging blood-based biomarkers for Alzheimer disease
}

$\mathrm{E}$

MERGING BLOOD-BASED BIOMARKERS for Alzheimer disease are an exciting new development in the dementia field, since they may offer a broadly accessible and relatively inexpensive screening tool. Looking to the future, when disease-modifying or prevention treatments will be available, investigators are focused on how to detect the earliest biological signals of Alzheimer disease, perhaps even years or decades before clinical symptoms appear.

The current standard workup for a patient with dementia symptoms focuses on disorders that may look like dementia or aggravate the early symptoms of Alzheimer disease, or a related dementia (eg, metabolic disorder, structural abnormality, vitamin deficiency). Currently, patients and their families want to know, Is this Alzheimer disease, or something that can be reversed? Current diagnostic testing can be challenging due to complexity, cost, or level of intervention. A validated blood test that could be widely utilized would be big step forward for diagnosing and, hopefully, intervening before a patient becomes clinically impaired.

\section{DEMENTIA'S TOLL}

An astonishing 5.8 million Americans age 65 and older have Alzheimer disease or a related dementia, and this number is expected to increase to 13.8 million by $2050 .{ }^{1}$

The impact on families is both financial and emotional. More than 16 million Americans currently provide unpaid care for family members or friends with dementia. The projected national cost of caring for those with Alzheimer disease and other dementias doi:10.3949/ccjm.87a.20133 is currently $\$ 305$ billion, which is unsustainable. As the aging population increases, so does the population with Alzheimer disease. The burden of caring for the increasing aging population with dementia is exacerbated by a shortage of dementia care specialists and the increasing burden on primary care clinicians to identify and provide care for these patients. ${ }^{1}$

\section{EARLY CHANGES IN THE BRAIN ARE HARD TO DETECT}

The pathologic hallmarks of Alzheimer disease are the accumulation in the brain of extracellular amyloid beta plaques and intraneuronal inclusions (neurofibrillary tangles) consisting of phosphorylated tau, a microtubule-associated protein. Also present are dystrophic neurites, loss of synapses, neuronal death, and gliosis. These pathologic changes can begin 10 to 20 years before the onset of clinical symptoms. ${ }^{2}$

Current validated biomarkers of Alzheimer disease pathology include:

- Amyloid beta and tau positron emission tomography (PET)

- The ratio of the concentrations in the cerebrospinal fluid of 2 amyloid beta peptides: the $1-42$ peptide and the $1-40$ peptide

- The concentrations of total tau and phosphorylated tau (specifically, phosphorylated at amino acid 181) in the cerebrospinal fluid. 3,4

The memory specialist is faced with a multitude of nuanced and mixed pathologies underlying a dementia syndrome. ${ }^{5}$ Biomarkers of Alzheimer disease pathology in combination with cognitive assessment and structural brain imaging can be valuable diagnostic tools in these circumstances. However, cerebrospinal
These

screening

tools could

help determine

who should

be referred

to a specialist

for in-depth

testing 
fluid analysis and PET are not easily utilized by the primary care clinician due to access, comfort with the testing or interpretation, and expense. Furthermore, the bedside cognitive testing currently used by primary care providers does not easily identify patients with early cognitive changes.

Therefore, for the primary care clinician, less-invasive and less-specialized screening tools, such as a blood test, would be a significant development. These screening tools could help determine who should be referred for more in-depth testing. Recent developments in the field are bringing us closer to blood tests that primary care clinicians can use as screening tools. This trend is promising, since it also will help in developing therapies targeting early-stage Alzheimer disease-specific pathology in larger and more diverse populations. Blood testing could fit into a diagnostic algorithm, similar to testing for certain cancers, that the primary care clinician could utilize for those at high risk of Alzheimer disease, such as the elderly and those with a strong family history.

\section{A blood test for Alzheimer disease could help in drug development and patient} care

\section{SEARCHING FOR A BLOOD-BASED BIOMARKER}

A major barrier to developing new drugs for Alzheimer disease is that it is hard to identify patients who are in the early stage of the disease, soon after the pathologic changes in the brain have begun but before cognitive impairment has become apparent, especially in the primary care setting. Given that an inexpensive and sensitive blood-based biomarker would enhance the ability of the primary care clinician to screen for possible Alzheimer disease, many researchers have focused significant effort on developing one.

\section{Circulating amyloid beta}

In early studies, plasma levels of amyloid beta lacked a consistent association with Alzheimer disease. ${ }^{6}$ This was most likely due to assayrelated difficulties, since plasma measurements of this protein may be influenced by matrix effects whereby other proteins in plasma bind it. However, later studies using more sensitive assays indicated that the plasma ratio of the amyloid beta $1-42$ and $1-40$ peptides was lower in amyloid PET-positive individuals, as it is in the cerebrospinal fluid, ${ }^{7-11}$ strongly suggesting that a plasma 1-42-to-1-40 ratio may be a feasible blood-based biomarker of Alzheimer disease. The only missing piece was a bloodbased measure of tau.

\section{Plasma total tau}

Initial studies of blood-based tau suggested that the plasma total tau concentration is higher in patients with Alzheimer disease than in cognitively normal controls. Unfortunately, the difference was not as clear or as well replicated as in cerebrospinal fluid. ${ }^{6}$ Subsequent studies also reported elevated plasma total tau in Alzheimer disease ${ }^{12,13}$ and an association with faster clinical disease progression, ${ }^{12}$ supporting the idea that plasma tau is indeed significantly elevated in Alzheimer disease.

\section{Plasma phosphorylated tau 181, tau 217}

Since cerebrospinal fluid phosphorylated tau 181, a key component of neurofibrillary tangles, adds better diagnostic accuracy than tau alone, researchers developed a new assay for phosphorylated tau at amino acid 181 in plasma. An association between this new phosphorylated tau 181 test and amyloid beta, as well as tau PET, was even stronger than those obtained using the plasma total tau test, ${ }^{14-16}$ strong evidence that plasma-phosphorylated tau 181 is a feasible blood-based biomarker of Alzheimer disease. However, since tau is phosphorylated at many sites, other phosphorylated sites may be better circulating biomarkers of Alzheimer disease. Most recently, intriguing new findings suggest that the plasma tau phosphorylated at amino acid 217 differs in patients with Alzheimer disease compared with cognitively normal controls and people with other neurodegenerative disorders. ${ }^{17,18}$ Plasma phosphorylated tau 217 is an intriguing finding, since it appears to outperform plasma phosphorylated tau 181 and imaging markers in terms of diagnostic accuracy. ${ }^{17,18}$

\section{STUDIES UNDER WAY}

While these new findings are encouraging, they are early results. These blood-based tests need further testing in large-scale studies over the long term to refine and verify them, especially in the general population.

There is as yet no gold standard biomarker for Alzheimer disease (or for vascular demen- 
tia) before clinical symptoms arise. Definitive diagnosis is done at autopsy, based on neuropathologic amyloid and tau findings.

The Alzheimer's Association Global Biomarker Standardization Consortium was established more than a decade ago to bring together key researchers, clinicians, industry, regulatory bodies, and government leaders in Alzheimer disease and other dementias. ${ }^{19}$ The goal is to achieve consensus on the best way to standardize and validate biomarker tests for use in clinical practice. Promising biomarkers have spurred several method-comparison and standardization studies across multiple laboratory sites, both nationally and internationally, under Consortium

\section{REFERENCES}

1. Alzheimer's Association. 2020 Alzheimer's disease facts and figures. Alzheimers Dement 2020 Mar 10. doi:10.1002/alz.12068

2. Gallardo G, Holtzman DM. Amyloid- $\beta$ and tau at the crossroads of Alzheimer's disease. Adv Exp Med Biol 2019; 1184:187203. doi:10.1007/978-981-32-9358-8 16

3. Cohen AD, Landau SM, Snitz BE, Klunk WE, Blennow K, Zetterberg $H$. Fluid and PET biomarkers for amyloid pathology in Alzheimer's disease. Mol Cell Neurosci 2019; 97:3-17. doi:10.1016/j.mcn.2018.12.004

4. Schöll M, Maass A, Mattsson N, et al. Biomarkers for tau pathology. Mol Cell Neurosci 2019; 97:18-33. doi:10.1016/j.mcn.2018.12.001

5. Boyle PA, Yu L, Leurgans SE, et al. Attributable risk of Alzheimer's dementia attributed to age-related neuropathologies. Ann Neurol 2019; 85(1):114-124. doi:10.1002/ana.25380

6. Olsson B, Lautner R, Andreasson U, et al. CSF and blood biomarkers for the diagnosis of Alzheimer's disease: a systematic review and meta-analysis. Lancet Neurol 2016; 15(7):673-684. doi:10.1016/S1474-4422(16)00070-3

7. Zetterberg H, Mörtberg E, Song L, et al. Hypoxia due to cardiac arrest induces a time-dependent increase in serum amyloid $B$ levels in humans. PloS One 2011; 6(12):e28263. doi:10.1371/journal.pone.0028263

8. Janelidze S, Stomrud E, Palmqvist S, et al. Plasma ß-amyloid in Alzheimer's disease and vascular disease. Sci Rep 2016; 6:26801. doi:10.1038/srep26801

9. Verberk IMW, Slot RE, Verfaillie SCJ, et al. Plasma amyloid as prescreener for the earliest Alzheimer pathological changes. Ann Neurol 2018; 84(5):648-658. doi:10.1002/ana.25334

10. Nakamura A, Kaneko N, Villemagne VL, et al. High performance plasma amyloid-ß biomarkers for Alzheimer's disease. Nature 2018; 554(7691):249-254. doi:10.1038/nature25456

11. Ovod V, Ramsey KN, Mawuenyega KG, et al. Amyloid B concentrations and stable isotope labeling kinetics of human plasma specific to central nervous system amyloidosis. Alzheimers Dement 2017; guidance. Future method-comparison and standardization studies will bring us closer to plasma amyloid and tau biomarkers as effective screenng tools in the primary care setting.

This is an exciting time, since blood-based biomarkers for Alzheimer disease are a potentially important step forward for both research and clinical care. As we move toward diseasemodifying therapies for Alzheimer disease and related dementias, they will be crucial for enhancing further clinical trial strategies, supporting primary care practice diagnosis and management, and, hopefully, moving to an era of better interventions for these devastating disorders.

13(8):841-849. doi:10.1016/j.jalz.2017.06.2266

12. Mielke MM, Hagen CE, Wennberg AMV, et al. Association of plasma total tau level with cognitive decline and risk of mild cognitive impairment or dementia in the Mayo Clinic Study on Aging. JAMA Neurol 2017; 74(9):1073-1080. doi:10.1001/jamaneurol.2017.1359

13. Pase MP, Beiser AS, Himali JJ, et al. Assessment of plasma total tau level as a predictive biomarker for dementia and related endophenotypes. JAMA Neurol 2019; 76(5):598-606. doi:10.1001/jamaneurol.2018.4666

14. Mielke MM, Hagen CE, Xu J, et al. Plasma phospho-tau181 increases with Alzheimer's disease clinical severity and is associated with tauand amyloid-positron emission tomography. Alzheimers Dement 2018; 14(8):989-997. doi:10.1016/j.jalz.2018.02.013

15. Yang C-C, Chiu M-J, Chen T-F, et al. Assay of plasma phosphorylated tau protein (threonine 181) and total tau protein in earlystage Alzheimer's disease. J Alzheimers Dis 2018; 61(4):1323-1332. doi:10.3233/JAD-170810

16. Tatebe H, Kasai T, Ohmichi T, et al. Quantification of plasma phosphorylated tau to use as a biomarker for brain Alzheimer pathology: pilot case-control studies including patients with Alzheimer's disease and Down syndrome. Mol Neurodegener 2017; 12(1):63. doi:10.1186/s13024-017-0206-8

17. Palmqvist $\mathbf{S}$, Janelidze $S$, Quiroz $\mathrm{YT}$, et al. Discriminative accuracy of plasma phospho-tau217 for Alzheimer disease vs other neurodegenerative disorders. JAMA 2020 Jul 28. doi:10.1001/jama.2020.12134

18. Barthélemy NR, Horie K, Sato C, Bateman RJ. Blood plasma phosphorylated-tau isoforms track CNS change in Alzheimer's disease. J Exp Med 2020; 217(11):e20200861. doi:10.1084/jem.20200861

19. Carrillo MC, Blennow K, Soares H, et al. Global standardization measurement of cerebral spinal fluid for Alzheimer's disease: an update from the Alzheimer's Association Global Biomarkers Consortium. Alzheimers Dement 2013; 9(2):137-140. doi:10.1016/j.jalz.2012.11.003

Address: Lynn M. Bekris, PhD, Genomic Medicine Institute, R4, Cleveland Clinic, 9500 Euclid Avenue, Cleveland, OH 44195; bekris/@ccf.org 